\title{
JOINTLY OPTIMAL DEREVERBERATION AND BEAMFORMING
}

\author{
Christoph Boeddeker ${ }^{1}$, Tomohiro Nakatani ${ }^{2}$, Keisuke Kinoshita ${ }^{2}$, Reinhold Haeb-Umbach ${ }^{1}$ \\ ${ }^{1}$ Paderborn University, Department of Communications Engineering, Paderborn, Germany \\ ${ }^{2}$ NTT Communication Science Laboratories, NTT Corporation, Kyoto, Japan
}

\begin{abstract}
We previously proposed an optimal (in the maximum likelihood sense) convolutional beamformer that can perform simultaneous denoising and dereverberation, and showed its superiority over the widely used cascade of a Weighted Prediction Error (WPE) dereverberation filter and a conventional Minimum-Power Distortionless Response (MPDR) beamformer. However, it has not been fully investigated which components in the convolutional beamformer yield such superiority. To this end, this paper presents a new derivation of the convolutional beamformer that allows us to factorize it into a WPE dereverberation filter, and a special type of a (nonconvolutional) beamformer, referred to as a weighted MPDR (wMPDR) beamformer, without loss of optimality. With experiments, we show that the superiority of the convolutional beamformer in fact comes from its wMPDR part.
\end{abstract}

Index Terms - Dereverberation, beamforming, speech enhancement

\section{INTRODUCTION}

In many speech processing applications the microphone signal is degraded both by reverberation and by noise. Reverberation is caused by the signal traveling from source to the sensor via multiple paths with different lengths and attenuations, causing a temporal smearing. While early reflections which arrive with up to roughly $50 \mathrm{~ms}$ delay compared to the direct signal are actually beneficial for human perception and even for Automatic Speech Recognition (ASR), late reverberation degrades both of them. Furthermore, if microphones are located at a distance to the speaker, it is likely that they capture other signals, here denoted as noise for simplicity, in addition to the desired speech signal.

For improving the quality of recorded speech, a number of techniques has been developed for dereverberation and denoising. Dereverberation techniques can be broadly categorized [1] into spectral magnitude manipulation [2] and linear filtering techniques [3, 4]. Among the latter, the WPE method [4, 5], has been shown to be very effective both for improving signal quality for human perception and for ASR [6]. A very effective mean to remove noise is acoustic beamforming based on microphone arrays [7, 8], which can enhance the desired source signal while attenuating signals with different propagation patterns. Furthermore, for performing dereverberation and denoising at the same time, their cascade configuration has been widely studied. Its effectiveness was shown by top scoring systems developed for recent distant speech recognition challenges, such as the REVERB and the CHiME-3/4/5 challenges $[6,9,10]$. Iterative optimization of the cascade configuration is also investigated as extension of this approach [11,12].

One issue of the conventional cascade approach $[12,13]$ was that the overall optimality was not guaranteed. The approach performs the optimization separately for dereverberation and beamforming. Moreover, different optimization criteria are adopted for the respective problems: The WPE technique estimates the dereverberation filter based on maximum likelihood estimation with a time-varying Gaussian source assumption [4], while most beamformers are estimated based on a noise power minimization criterion [7]. As a consequence, what is optimized by the cascaded approach was even not clear. Also, it was not well investigated what optimization criterion is preferable for simultaneous denoising and dereverberation.

To address this issue, a convolutional beamformer has been recently proposed [14]. It unifies the WPE dereverberation filter and a beamformer into a single linear convolutional filter, called weighted power minimization distortionless response (WPD) beamformer. The filter coefficients are optimized based on a single criterion, namely the likelihood maximization with a time-varying Gaussian source assumption [15]. The WPD beamformer was compared with a conventional cascade configuration, consisting of a WPE Multiple Input Multiple Output (MIMO) dereverberation filter [5] followed by an MPDR beamformer. Experiments carried out on the REVERB challenge data set showed a performance advantage in terms of word error rate (WER) of the WPD over the cascade structure.

However, it remains unclear what makes the WPD beamformer superior to the conventional cascade configuration, and if at all there is an essential difference between the unified and cascade structures. This paper answers these questions and shows the equivalence between the WPD convolutional beamforming and the cascade configuration of MIMO-WPE dereverberation followed by a variant of MPDR beamforming, namely weighted MPDR (wMPDR) beamforming. We theoretically derive their strict equivalence under the assumption that the two are jointly optimized based on the maximum likelihood criterion. The factorizability of the convolutional beamforming has some practical advantages due to its modularity. For example, signal parameters, such as the spatial covariance matrix of the vector of microphone signals and the time variant clean speech power, need to be estimated from the data. In [15], this was done with the help of additional WPE preprocessing. With the result given here, this WPE component can be a part of the enhancement chain, thus simplifying the overall structure.

We further show that the performance advantage obtained by the WPD beamforming or its equivalent cascaded structure over the conventional cascade structure comes from the wMPDR beamforming over conventional beamforming.

The paper is organized as follows: After the signal model is introduced in Sec. 2, unified and factorized versions of the convolutional beamformer are derived in Secs. 3 and 4. Sec. 5 discusses the characteristics of the factorized and unifed structure referring to its equivalence shown in the Appendix. Experimental validation of the theory and concluding remarks are given in Secs. 6 and 7. 


\section{SIGNAL MODEL}

We assume that a single speech signal is captured by $M$ microphones in a noisy and reverberant environment. In the Short Time Fourier Transform (STFT) domain the vector of microphone signals $\mathbf{y}_{t}=$ $\left[\begin{array}{lll}y_{1, t} & \ldots & y_{M, t}\end{array}\right]^{\mathrm{T}}$ can be written as the convolution of the source signal $s_{t}$ with the vector of the convolutive transfer function $\mathbf{a}_{\tau}=$ $\left[\begin{array}{lll}a_{1, \tau} & \ldots & a_{M, \tau}\end{array}\right]^{\mathrm{T}}$ plus an additive noise vector $\mathbf{n}_{t}$ :

$$
\begin{aligned}
\mathbf{y}_{t} & =\sum_{\tau=0}^{L_{a}-1} \mathbf{a}_{\tau} s_{t-\tau}+\mathbf{n}_{t} \\
& =\mathbf{x}_{t}+\mathbf{n}_{t}=\mathbf{d}_{t}+\mathbf{r}_{t}+\mathbf{n}_{t} .
\end{aligned}
$$

Here, $t$ is the time frame index. The frequency bin index has been dropped for ease of notation. $L_{a}$ is the length of the transfer function in number of frames. The term $\mathbf{x}_{t}$ is called the image of the source signal $s_{t}$ at the microphones, which is further decomposed in the direct signal plus early reflections $\mathbf{d}_{t}$, and late reverberation $\mathbf{r}_{t}$ :

$$
\begin{aligned}
\mathbf{d}_{t} & =\sum_{\tau=0}^{b-1} \mathbf{a}_{\tau} s_{t-\tau} \approx \mathbf{v} s_{t}=\tilde{\mathbf{v}} d_{1, t} \\
\mathbf{r}_{t} & =\sum_{\tau=b}^{L_{a}-1} \mathbf{a}_{\tau} s_{t-\tau},
\end{aligned}
$$

where the frame index $b$ separates the early reflections from the late reverberation. A typical value for $b$ is 2 to 4 frames, corresponding to 30 to $50 \mathrm{~ms}$. In (3) we approximated $\mathbf{d}_{t}$ by the product of a timeinvariant (non-convolutive) acoustic transfer function (ATF) vector $\mathbf{v}$ with the clean speech signal $s_{t}$. Furthermore we introduced the relative transfer function (RTF) $\tilde{\mathbf{v}}=\mathbf{v} / v_{1}$, and $d_{1, t}=v_{1} s_{t}$.

We now define the vector of the past $L_{w}$ microphone signals, including the current observation $\mathbf{y}_{t}$, but excluding the most recent $b-1$ frames:

$$
\begin{aligned}
\overline{\mathbf{y}}_{t} & =\left[\begin{array}{llll}
\mathbf{y}_{t}^{\mathrm{T}} & \mathbf{y}_{t-b}^{\mathrm{T}} & \cdots & \mathbf{y}_{t-L_{w}+1}^{\mathrm{T}}
\end{array}\right]^{\mathrm{T}} \in \mathbb{C}^{M\left(L_{w}-b+1\right) \times 1} \\
& =\left[\begin{array}{ll}
\mathbf{y}_{t}^{\mathrm{T}} & \tilde{\mathbf{y}}_{t}^{\mathrm{T}}
\end{array}\right]^{\mathrm{T}}
\end{aligned}
$$

where $\tilde{\mathbf{y}}$ captures the observations from $b$ frames in the past until $L_{w}-1$ frames in the past. Our goal is to determine the coefficients $\overline{\mathbf{w}}$ of a spatial filter such that

$$
z_{t}=\overline{\mathbf{w}}^{\mathrm{H}} \overline{\mathbf{y}}_{t}
$$

is an estimate of the desired signal $d_{1, t}$. Here, $\overline{\mathbf{w}}$ is the vector

$$
\overline{\mathbf{w}}=\left[\begin{array}{llll}
\mathbf{w}_{0}^{\mathrm{T}} & \mathbf{w}_{b}^{\mathrm{T}} & \ldots & \mathbf{w}_{L_{w}-1}^{\mathrm{T}}
\end{array}\right]^{\mathrm{T}},
$$

which has the same dimension as $\overline{\mathbf{y}}$. Because this beamformer is based on the convolutional signal model of eq. (1) we call it convolutional beamformer [14].

\section{UNIFIED SOLUTION}

In [15], the output $z_{t}$ is modeled as a zero mean complex Gaussian with a time varying variance. This output distribution was used to define the maximum likelihood (ML) objective for the estimation of the coefficients $\overline{\mathbf{w}}$. Under a distortionless response constraint that is often introduced into beamforming the ML objective can be replaced with:

$$
\begin{aligned}
\mathcal{L}(\overline{\mathbf{w}}) & \propto \frac{1}{T} \sum_{t=1}^{T}\left(-\ln \left(\lambda_{t}\right)-\frac{\left|z_{t}\right|^{2}}{\lambda_{t}}\right) \\
& \propto-\overline{\mathbf{w}}^{\mathrm{H}}\left(\frac{1}{T} \sum_{t=1}^{T} \frac{\overline{\mathbf{y}}_{t} \overline{\mathbf{y}}_{t}^{\mathrm{H}}}{\lambda_{t}}\right) \overline{\mathbf{w}}=-\overline{\mathbf{w}}^{\mathrm{H}} \overline{\mathbf{R}}_{\mathbf{y}} \overline{\mathbf{w}},
\end{aligned}
$$

where $\lambda_{t}=\mathbb{E}\left[\left|d_{1, t}\right|^{2}\right], \overline{\mathbf{R}}_{\mathbf{y}}=\frac{1}{T} \sum_{t} \overline{\mathbf{y}}_{t} \overline{\mathbf{y}}_{t}^{\mathrm{H}} / \lambda_{t}$, and where $T$ is the number of frames over which the beamformer coefficients are estimated. The distortionless response constraint introduced for the ML estimation was:

$$
\mathbf{w}_{0}^{\mathrm{H}} \tilde{\mathbf{v}}=1 .
$$

This can be reformulated by introducing $\overline{\mathbf{w}}$ as follows:

$$
\overline{\mathbf{w}}^{\mathrm{H}} \overline{\mathbf{v}}=1,
$$

where $\overline{\mathbf{v}}=\left[\begin{array}{ll}\mathbf{v}^{\mathrm{T}} / v_{1} & \mathbf{0}^{\mathrm{T}}\end{array}\right]^{\mathrm{T}}$, and where $\mathbf{0}$ is a vector of zeros of dimension $\left(M \cdot\left(L_{w}-b\right) \times 1\right)$. A constrained optimization problem

$$
\mathcal{L}(\overline{\mathbf{w}})=-\overline{\mathbf{w}}^{\mathrm{H}} \overline{\mathbf{R}}_{\mathbf{y}} \overline{\mathbf{w}} \quad \text { s.t. } \quad \overline{\mathbf{w}}^{\mathrm{H}} \overline{\mathbf{v}}=1
$$

of this kind is well-known from minimum variance/power distortionless beamforming, and the solution is given by

$$
\overline{\mathbf{w}}=\frac{\overline{\mathbf{R}}_{\mathbf{y}}^{-1} \overline{\mathbf{v}}}{\overline{\mathbf{v}}^{\mathrm{H}} \overline{\mathbf{R}}_{\mathbf{y}}^{-1} \overline{\mathbf{v}}} .
$$

This is the WPD beamformer proposed in [14].

\section{FACTORIZED SOLUTION}

Now we assume that $\overline{\mathbf{w}}$ factorizes into a $\left(M\left(L_{w}-b+1\right) \times M\right)$ dimensional MIMO dereverberation matrix $\overline{\mathbf{G}}$ and a beamforming vector $\mathbf{q}$ of size $(M \times 1)$

$$
\overline{\mathbf{w}}=\overline{\mathbf{G}} \mathbf{q} .
$$

Using this in the objective function (10) we obtain

$$
\mathcal{L}(\overline{\mathbf{G}}, \mathbf{q}) \propto-\mathbf{q}^{\mathrm{H}} \overline{\mathbf{G}}^{\mathrm{H}} \overline{\mathbf{R}}_{\mathbf{y}} \overline{\mathbf{G}} \mathbf{q} .
$$

Note that $\overline{\mathbf{G}}$ has a particular structure, because we have to make sure that the direct signal and early reflections remain unmodified by the derevereberation matrix:

$$
\overline{\mathbf{G}}=\left[\begin{array}{c}
\mathbf{I}_{M} \\
-\tilde{\mathbf{G}}
\end{array}\right]
$$

Here, $\mathbf{I}_{M}$ is the $(M \times M)$-dimensional identity matrix, and $\tilde{\mathbf{G}}$ is of dimension $\left(M\left(L_{w}-b\right) \times M\right)$. Similarly, we factorize $\overline{\mathbf{R}}_{\mathbf{y}}$ :

$$
\overline{\mathbf{R}}_{\mathbf{y}}=\left[\begin{array}{ll}
\mathbf{R}_{\mathbf{y}} & \mathbf{P}_{\mathbf{y}}^{\mathrm{H}} \\
\mathbf{P}_{\mathbf{y}} & \tilde{\mathbf{R}}_{\mathbf{y}}
\end{array}\right]
$$

where $\mathbf{R}_{\mathbf{y}}=\frac{1}{T} \sum_{t=1}^{T} \mathbf{y}_{t} \mathbf{y}_{t}^{\mathrm{H}} / \lambda_{t}, \mathbf{P}_{\mathbf{y}}=\frac{1}{T} \sum_{t=1}^{T} \tilde{\mathbf{y}}_{t} \mathbf{y}_{t}^{\mathrm{H}} / \lambda_{t}$, and $\tilde{\mathbf{R}}_{\mathbf{y}}=\frac{1}{T} \sum_{t=1}^{T} \tilde{\mathbf{y}}_{t} \tilde{\mathbf{y}}_{t}^{\mathrm{H}} / \lambda_{t}$. Using this in (16), we arive at

$$
\begin{aligned}
& \mathcal{L}(\tilde{\mathbf{G}}, \mathbf{q}) \propto-\mathbf{q}^{\mathrm{H}}\left[\begin{array}{c}
\mathbf{I} \\
-\tilde{\mathbf{G}}
\end{array}\right]^{\mathrm{H}}\left[\begin{array}{ll}
\mathbf{R}_{\mathbf{y}} & \mathbf{P}_{\mathbf{y}}^{\mathrm{H}} \\
\mathbf{P}_{\mathbf{y}} & \tilde{\mathbf{R}}_{\mathbf{y}}
\end{array}\right]\left[\begin{array}{c}
\mathbf{I} \\
-\tilde{\mathbf{G}}
\end{array}\right] \mathbf{q} \\
& =-\mathbf{q} \mathbf{R}_{\mathbf{y}} \mathbf{q}+\mathbf{q}^{\mathrm{H}} \tilde{\mathbf{G}}^{\mathrm{H}} \mathbf{P}_{\mathbf{y}} \mathbf{q}+\mathbf{q}^{\mathrm{H}} \mathbf{P}_{\mathbf{y}}^{\mathrm{H}} \tilde{\mathbf{G}} \mathbf{q}-\mathbf{q}^{\mathrm{H}} \tilde{\mathbf{G}}^{\mathrm{H}} \tilde{\mathbf{R}} \mathbf{y} \tilde{\mathbf{G}} \mathbf{q} .
\end{aligned}
$$

To calculate the derivative w.r.t. the dereverberation matrix we use eq. (70), (71) and (82) from [16] and the property $\tilde{\mathbf{R}}_{\mathbf{y}}=\tilde{\mathbf{R}}_{\mathbf{y}}^{\mathrm{H}}$. Setting the derivative to zero gives

$$
\frac{\partial \mathcal{L}(\tilde{\mathbf{G}}, \mathbf{q})}{\partial \tilde{\mathbf{G}}}=2 \mathbf{P}_{\mathbf{y}} \mathbf{q} \mathbf{q}^{\mathrm{H}}-2 \tilde{\mathbf{R}}_{\mathbf{y}} \tilde{\mathbf{G}} \mathbf{q} \mathbf{q}^{\mathrm{H}} \stackrel{!}{=} \mathbf{0} .
$$

This equation has obviously multiple solutions. A solution, which allows separate estimation of $\tilde{\mathbf{G}}$ and $\mathbf{q}$ is

$$
\tilde{\mathbf{G}}=\tilde{\mathbf{R}}_{\mathbf{y}}^{-1} \mathbf{P}_{\mathbf{y}} .
$$


This solution is identical to the WPE solution [5, 17, 18] (Scaled Identity Matrix assumption: $\left.\breve{\mathbf{d}} \sim \mathcal{N}\left(\mathbf{0}, \lambda_{t} \mathbf{I}\right)\right)$. It allows to obtain a first estimate the dereverberated signal from the current observation via

$$
\breve{\mathbf{d}}_{t}=\overline{\mathbf{G}}^{\mathrm{H}} \overline{\mathbf{y}}_{t} .
$$

Next we optimize (16) w.r.t. the beamforming vector. This is analog to eq. (13), if $\overline{\mathbf{w}}$ and $\overline{\mathbf{R}}_{\mathbf{y}}$ are replaced by $\mathbf{q}$ and $\mathbf{R}_{\mathbf{d}}=\overline{\mathbf{G}}^{\mathrm{H}} \overline{\mathbf{R}}_{\mathbf{y}} \overline{\mathbf{G}}$, respectively. Thus, using the constraint $\mathbf{q}^{\mathrm{H}} \tilde{\mathbf{v}}=1$ gives the solution:

$$
\mathbf{q}=\frac{\mathbf{R}_{\mathbf{d}}^{-1} \tilde{\mathbf{v}}}{\tilde{\mathbf{v}}^{\mathrm{H}} \mathbf{R}_{\mathbf{d}}^{-1} \tilde{\mathbf{v}}} .
$$

$\mathbf{R}_{\mathbf{d}}$ can be estimated just like we estimated $\overline{\mathbf{R}}_{\mathbf{y}}$ above:

$$
\mathbf{R}_{\mathbf{d}}=\frac{1}{T} \sum_{t} \overline{\mathbf{G}}^{\mathrm{H}} \frac{\overline{\mathbf{y}}_{t} \overline{\mathbf{y}}_{t}^{\mathrm{H}}}{\lambda_{t}} \overline{\mathbf{G}}=\frac{1}{T} \sum_{t} \frac{\breve{\mathbf{d}}_{t} \breve{\mathbf{d}}_{t}^{\mathrm{H}}}{\lambda_{t}} .
$$

This beamformer is similar to the MPDR beamformer, but the denominator in eq. (24) makes this beamformer a wMPDR. It is worth noting that this beamformer can be derived as a special case of WPD by assuming the absence of reverberation and setting the length of the convolutional beamformer in (1)-(14) to $L_{w}=1$. This beamformer was independently proposed as a Maximum Likelihood Distortionless Response (MLDR) in [19].

\section{DISCUSSION}

In the appendix we show that the unified (WPD) beamformer and factorized solution, which consists of the cascade of WPE dereverberation and wMPDR beamforming, are identical. Instead of estimating the coefficient vector of the convolutional beamformer, it is thus equivalent to first dereverberate the vector of microphone signals using the MIMO-WPE method and then applying a wMPDR beamformer resting on the narrowband assumption to the result. This cascaded solution may have some practical advantages, because it allows to treat dereverberation and beamforming separately. Although the equivalence has only been derived for the ML convolutional beamformer, it may still be seen as an indication, that a cascade of dereverberation with a beamformer optimized under another criterion is a legitimate solution as done in [12].

Comparing the constrained optimization problem (13) with the classical MPDR, the difference is the scaling of the beamformer output power by the variance of the clean speech signal. This scaling in the objective function accounts for the time-varying nature of the speech power. Observations with large speech variance are downscaled, while observations with a low variance are emphasized for spatial covariance estimation for beamforming coefficient computation. This makes sense because we do not want to destroy the speech signal and only suppress the distortions.

In practice the parameters of the statistical models involved have to be estimated from the data. This includes the RTF $\tilde{\mathbf{v}}$ and the timevariant power spectral density of the desired speech component $\lambda_{t}$, which will be discussed in the next section.

\section{EXPERIMENTS}

In this section, we experimentally confirm the equivalence of the unified and factorized solutions, and present detailed analysis of the factorized solution.

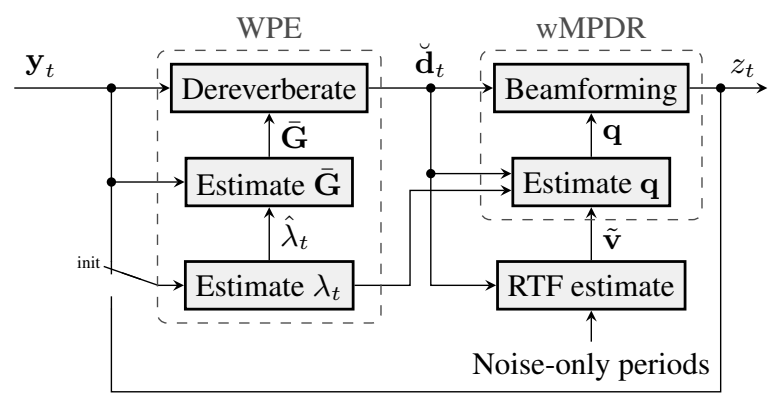

Fig. 1: Proposed factorisation of WPD in WPE and wMPDR with RTF estimation and power estimation (joint optimization).

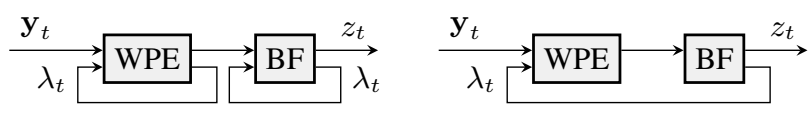

(a) Separate optimization

(b) Joint optimization

Fig. 2: Separate and joint optimization schemes.

\subsection{Equivalence experiment}

We first show the equivalence of the unified and factorized solution experimentally by applying both to a CHiME3 utterance [9]. The RTF is estimated with the help of oracle energy ratio masks (Wiener like [8]), and the Power Spectral Density (PSD) of the source, $\lambda_{t}$, is determined as the power of the observation. Using these values we obtain an estimate for the factorized solution $z_{t}^{\text {factorized }}$ and for the unified solution $z_{t}^{\text {unified }}$ for each frequency. Then, to verify the equivalence, we tested that the following inequality holds for every time-frequency point in the STFT:

$$
\frac{\left\|z_{t}^{\text {factorized }}-z_{t}^{\text {unified }}\right\|}{\left\|z_{\tilde{t}}^{\text {factorized }}\right\|} \leq 10^{-9}
$$

A maximum relative difference of $10^{-9}$ is reasonable for doubleprecision floating-point values, where different mathematical calculations are used (e.g. in both solutions a linear system of equations has to be solved, but in the unified solution there are more linear equations: (14) vs (21)).

\subsection{Experimental analysis of proposed factorization}

In the following, we present an experimental analysis of the proposed factorization (WPE+wMPDR) by comparing it with the conventional cascade configuration (WPE+MPDR) and various beamforming configurations, including MPDR, MVDR, and wMPDR.

\subsubsection{Dataset, evaluation metrics, and analysis conditions}

For the analysis, we used the REVERB Challenge dataset (REVERB) [6] and the CHiME3 challenge dataset (CHiME3) [9]. Each utterance in REVERB was recorded in reverberant environments with a little stationary additive noise, while that in CHiME3 was recorded in public areas with relatively high level non-stationary ambient noise and a little reverberation. Separate optimization of WPE and MVDR/MPDR has been shown to be very effective as frontend of ASR for both dataset $[6,9]$.

For the performance evaluation, we used baseline ASR systems recently developed using Kaldi [20], respectively, for REVERB and CHiME3. They are fairly competitive systems composed of a TDNN 
Table 1: WERs (\%) of enhanced speech obtained after 1st iteration. Boldface indicates the best score for each condition.

\begin{tabular}{ccc}
\hline & \multicolumn{2}{c}{ Real eval set } \\
\cline { 2 - 3 } & CHiME3 & REVERB \\
\hline Obs & 17.83 & 18.61 \\
MPDR & 7.47 & 13.14 \\
MVDR & 7.50 & 12.87 \\
wMPDR & $\mathbf{6 . 9 9}$ & $\mathbf{1 2 . 6 5}$ \\
\hline WPE & 13.95 & 13.24 \\
\hline WPE+MPDR (joint opt.) & 7.55 & 10.06 \\
WPE+wMPDR (joint opt.) & $\mathbf{7 . 0 7}$ & $\mathbf{9 . 5 2}$ \\
\hline
\end{tabular}

acoustic model trained using lattice-free MMI, online i-vector extraction, and a trigram language model.

A Hann window was used for a short-time analysis with the sampling frequency being $16 \mathrm{kHz} . M=8$ and $M=6$ microphones were used, respectively, for REVERB and CHiME3. The prediction delay was set at $b=4$, and the length of the prediction filter was set at $L_{w}=12,10$, and 6 , respectively, for frequency ranges of 0 to 0.8 $\mathrm{kHz}, 0.8$ to $1.5 \mathrm{kHz}, 1.5$ to $8 \mathrm{kHz}$, for REVERB, and $L_{w}=7$ for CHiME3.

\subsubsection{Estimation of power spectral density and RTF}

Figure 1 illustrates the overall processing flow of the estimation, where we jointly estimate the PSD, $\lambda_{t}$, and the RTF, $\tilde{\mathbf{v}}$. The PSD $\lambda_{t}$ is estimated with the same ML objective, but since no closed-form solution is known, the PSD $\lambda_{t}$ and the convolutional beamformer are estimated alternatingly based on iterative optimization [15]. Maximizing (9) w.r.t. $\lambda_{t}$ will yield

$$
\lambda_{t}=\left|z_{t}\right|^{2}=\left|\overline{\mathbf{w}}^{\mathrm{H}} \overline{\mathbf{y}}_{t}\right|^{2} .
$$

This parameter estimation is referred to as joint optimization scheme shown in fig. 2b. In the experiments, we also test a separate optimization scheme shown in (fig. 2a), where the WPE and beamforming are optimized separately and the PSD is estimated using iterative optimization of respective processing blocks.

For the estimation of the RTF $\tilde{\mathbf{v}}$, we used a method based on eigenvalue decomposition with noise covariance whitening [21, 22], and apply it to the output of WPE dereverberation, to reduce the effect of reverberation and noise from the estimation. For estimation of noise spatial covariance matrices, we assumed that each utterance had noise-only periods of $225 \mathrm{~ms}$ and $75 \mathrm{~ms}$, respectively, at its beginning and ending parts, for REVERB, and we used noise masks estimated by a BLSTM network [23] for CHiME3.

\subsubsection{Evaluation results}

Table 1 summarizes the WERs of the observed signals (Obs) and the enhanced signals obtained after the first estimation iteration. Here, we used the joint optimization scheme for WPE+wMPDR. In the table, WPE+wMPDR was the best among all the methods. When we compare wMPDR with MPDR/MVDR, and compare WPE+wMPDR with WPE+MPDR, wMPDR and WPE+wMPDR consistently outperformed MPDR/MVDR and WPE+MPDR, respectively. This shows that the superiority of the convolutional beamformer is surely derived from the superiority of wMPDR embedded into it.

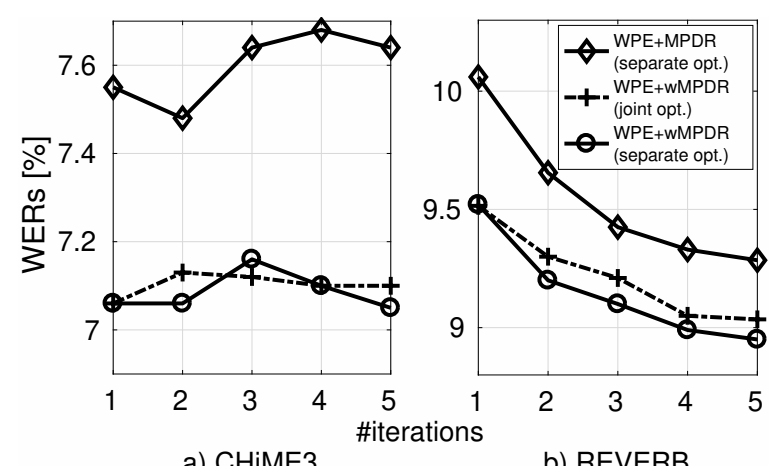

a) $\mathrm{CHIME3}$

b) REVERB

Fig. 3: WERs (\%) obtained with \# of estimation iterations by WPE+MPDR and WPE+wMPDR with joint and separate optimization schemes

Figure 3 shows the WERs obtained by iterative estimation. In addition to the joint optimization scheme for the estimation of $\lambda_{t}$, we used the separate optimization scheme. With the separate optimization, a specified number of iterations is first performed by WPE and then performed by beamforming. We test this configuration as a simpler alternative of WPE+wMPDR. As shown in the figure, with both joint and separate optimization schemes, the proposed factorization, WPE+wMPDR, achieved almost the same WERs, and they are consistently better than the conventional cascade configuration, WPE+MPDR, with the separate optimization.

\section{CONCLUSIONS}

In this contribution we factorized the WPD convolutional beamformer in WPE dereverberation and wMPDR beamforming and displayed practical advantages. The equivalence is verified mathematically and numerically. In experiments on real data we showed that the strength of the WPD convolutional beamformer has its origin in the wMPDR beamformer. A comparison of a simple separate optimization with a joint optimization of WPE and wMPDR yielded similar WERs.

\section{APPENDIX: UNIFIED VERSUS FACTORIZED SOLUTION}

We now show that the two solutions, Sec. 3 and Sec. 4 , are identical. Starting with the factorized solution, note first that $\mathbf{R}_{\mathbf{d}}=\overline{\mathbf{G}}^{\mathrm{H}} \overline{\mathbf{R}}_{\mathbf{y}} \overline{\mathbf{G}}$ can be expressed as

$$
\mathbf{R}_{\mathbf{d}}=\left(\mathbf{R}_{\mathbf{y}}-\mathbf{P}_{\mathbf{y}}^{\mathrm{H}} \tilde{\mathbf{R}}_{\mathbf{y}}^{-1} \mathbf{P}_{\mathbf{y}}\right)
$$

using (17), (18) and (21). Employing this in (15) we can express the convolutional beamformer coefficients as

$$
\begin{aligned}
\overline{\mathbf{w}} & =\overline{\mathbf{G}} \mathbf{q} \\
& =\left[\begin{array}{c}
\mathbf{I} \\
-\tilde{\mathbf{R}}_{\mathbf{y}}^{-1} \mathbf{P}_{\mathbf{y}}
\end{array}\right] \frac{\left(\mathbf{R}_{\mathbf{y}}-\mathbf{P}_{\mathbf{y}}^{\mathrm{H}} \tilde{\mathbf{R}}_{\mathbf{y}}^{-1} \mathbf{P}_{\mathbf{y}}\right)^{-1} \tilde{\mathbf{v}}}{\tilde{\mathbf{v}}^{\mathrm{H}}\left(\mathbf{R}_{\mathbf{y}}-\mathbf{P}_{\mathbf{y}}^{\mathrm{H}} \tilde{\mathbf{R}}_{\mathbf{y}}^{-1} \mathbf{P}_{\mathbf{y}}\right)^{-1} \tilde{\mathbf{v}}}
\end{aligned}
$$

where we expressed $\overline{\mathbf{G}}$ using (17) and (21), and q using (23).

On the other hand, we take the unified solution (14) and plug in the definition of $\overline{\mathbf{R}}_{\mathbf{y}}$, eq. (18). Employing the the $(2 \times 2)$ block matrix inversion rule, we exactly obtain (28). Thus, the solutions (14) and (28) are identical! 


\section{REFERENCES}

[1] J. Li, L. Deng, R. Haeb-Umbach, and Y. Gong, Robust Automatic Speech Recognition; ch. 9: Reverberant Speech Recognition, Elsevier, Oct 2015.

[2] K. Lebart, J. M. Boucher, and P. Denbigh, "A new method based on spectral subtraction for speech dereverberation," Acta Acoustica, vol. 87, no. 3, pp. 359366, 2001.

[3] O. Schwartz, S. Gannot, and E. A. P. Habets, "Multimicrophone speech dereverberation and noise reduction using relative early transfer function," IEEE/ACM Trans. Audio, Speech, Lang. Process., vol. 23, no. 2, pp. 240-251, 2015.

[4] T. Nakatani, T. Yoshioka, K. Kinoshita, M. Miyoshi, and B.-H. Juang, "Speech dereverberation based on variance-normalized delayed linear prediction," IEEE/ACM Trans. Audio, Speech, Lang. Process., vol. 18, no. 7, pp. 1717-1731, 2010.

[5] T. Yoshioka and T. Nakatani, "Generalization of multi-channel linear prediction methods for blind MIMO impulse response shortening," IEEE/ACM Trans. Audio, Speech, Lang. Process., 2012.

[6] K. Kinoshita, M. Delcroix, S. Gannot, E. Habets, R. HaebUmbach, W. Kellermann, V. Leutnant, R. Maas, T. Nakatani, B. Raj, A. Sehr, and T. Yoshioka, "A summary of the REVERB challenge: state-of-the-art and remaining challenges in reverberant speech processing research," EURASIP Journal on Advances in Signal Processing, 2016.

[7] B. D. Van Veen and K. M. Buckley, "Beamforming: A versatile approach to spatial filtering," IEEE ASSP Magazine, vol. 5, no. 2, pp. 4-24, April 1988.

[8] H. Erdogan, J. R. Hershey, S. Watanabe, and J. Le Roux, "Phase-sensitive and recognition-boosted speech separation using deep recurrent neural networks," in 2015 IEEE International Conference on Acoustics, Speech and Signal Processing (ICASSP). IEEE, 2015, pp. 708-712.

[9] J. Barker, R. Marxer, E. Vincent, and S. Watanabe, "The third CHiME speech separation and recognition challenge: Dataset, task and baselines," in 2015 IEEE Workshop on Automatic Speech Recognition and Understanding (ASRU). IEEE, 2015, pp. 504-511.

[10] N. Kanda, C. Boeddeker, J. Heitkaemper, Y. Fujita, S. Horiguchi, K. Nagamatsu, and R. Haeb-Umbach, "Guided source separation meets a strong ASR backend: Hitachi/Paderborn University joint investigation for dinner party ASR," in Proc. Interspeech, Sep 2019.

[11] S. Braun and E. A. P. Habets, "Linear prediction-based online dereverberation and noise reduction using alternating Kalman filter," IEEE/ACM Trans. Audio, Speech, Lang. Process., vol. 28, no. 6, pp. 1119-1129, 2018.

[12] L. Drude, C. Boeddeker, J. Heymann, R. Haeb-Umbach, K. Kinoshita, M. Delcroix, and T. Nakatani, "Integrating neural network based beamforming and weighted prediction error dereverberation.," in Interspeech, 2018, pp. 3043-3047.
[13] M. Delcroix, T. Yoshioka, A. Ogawa, Y. Kubo, M. Fujimoto, I. Nobutaka, K. Kinoshita, M. Espi, T. Hori, and T. Nakatani, "Strategies for distant speech recognition in reverberant environments," EURASIP Journal on Advances in Signal Processing, 2015.

[14] T. Nakatani and K. Kinoshita, "A unified convolutional beamformer for simultaneous denoising and dereverberation," IEEE Signal Processing Letters, vol. 26, pp. 903-907, April 2019.

[15] T. Nakatani and K. Kinoshita, "Maximum likelihood convolutional beamformer for simultaneous denoising and dereverberation," in Proc. EUSIPCO, A Coruna, Spain, Sep. 2019.

[16] K. B. Petersen, M. S. Pedersen, et al., "The matrix cookbook," Technical University of Denmark, vol. 7, no. 15, pp. 510, 2008.

[17] L. Drude, J. Heymann, C. Boeddeker, and R. Haeb-Umbach, "NARA-WPE: A Python package for weighted prediction error dereverberation in Numpy and Tensorflow for online and offline processing," in Speech Communication; 13th ITGSymposium. VDE, 2018, pp. 1-5.

[18] C. Boeddeker, L. Drude, and R. Haeb-Umbach, "Optimization of multi-channel dereverberation techniques for noisy reverberant speech recognition," M.S. thesis, Paderborn University, 2018.

[19] B. J. Cho, J. Lee, and H. Park, "A beamforming algorithm based on maximum likelihood of a complex Gaussian distribution with time-varying variances for robust speech recognition," IEEE Signal Processing Letters, vol. 26, no. 9, pp. 1398-1402, Sep. 2019.

[20] D. Povey, A. Ghoshal, G. Boulianne, L. Burget, O. Glembek, N. Goel, M. Hannemann, P. Motlicek, Y. Qian, P. Schwarz, J. Silovsky, G. Stemmer, and K. Vesely, "The Kaldi speech recognition toolkit," in IEEE 2011 Workshop on Automatic Speech Recognition and Understanding. Dec. 2011, IEEE Signal Processing Society, IEEE Catalog No.: CFP11SRW-USB.

[21] N. Ito, S. Araki, M. Delcroix, and T. Nakatani, "Probabilistic spatial dictionary based online adaptive beamforming for meeting recognition in noisy and reverberant environments," in 2017 IEEE International Conference on Acoustics, Speech and Signal Processing (ICASSP).

[22] S. Markovich-Golan and S. Gannot, "Performance analysis of the covariance subtraction method for relative transfer function estimation and comparison to the covariance whitening method," in 2015 IEEE International Conference on Acoustics, Speech and Signal Processing (ICASSP). IEEE, 2015, pp. 544-548.

[23] J. Heymann, L. Drude, C. Boeddeker, P. Hanebrink, and R. Haeb-Umbach, "Beamnet: End-to-end training of a beamformer-supported multi-channel ASR system," in 2017 IEEE International Conference on Acoustics, Speech and Signal Processing (ICASSP). IEEE, 2017, pp. 5325-5329. 